\title{
Multi-jet merging with NLO matrix elements
}

\author{
Frank Siegert ${ }^{* \dagger}$ \\ Physikalisches Institut, Albert-Ludwigs-Universität Freiburg \\ E-mail: frank.siegertecern.ch
}

\section{Stefan Höche}

SLAC National Accelerator Laboratory, Stanford University

E-mail: shoecheeslac.stanford.edu

\section{Frank Krauss}

IPPP, Durham University

E-mail: frank.krauss@durham.ac.uk

\section{Marek Schönherr}

Institut für Kern- und Teilchenphysik, Technische Universität Dresden

E-mail: marek.schoenherr@tu-dresden.de

In the algorithm presented here, the ME+PS approach [1] to merge samples of tree-level matrix elements into inclusive event samples is combined with the POWHEG method [2], which includes exact next-to-leading order matrix elements in the parton shower. The advantages of the method are discussed and the quality of its implementation in SHERPA is exemplified by results for $e^{+} e^{-}$ annihilation into hadrons at LEP, for deep-inelastic lepton-nucleon scattering at HERA, for DrellYan lepton-pair production at the Tevatron and for $W^{+} W^{-}$-production at LHC energies.

35th International Conference of High Energy Physics

July 22-28, 2010

Paris, France

\footnotetext{
*Speaker.

${ }^{\dagger}$ Supported by the MCnet Marie Curie Research Training Network MRTN-CT-2006-035606.
} 


\section{Introduction}

The simulation of hard QCD radiation in parton-shower Monte Carlos has seen tremendous progress over the last years. It was largely stimulated by the need for more precise predictions at LHC energies where the large available phase space allows additional hard QCD radiation alongside known Standard Model processes or even signals from new physics.

Two types of algorithms have been developed, which allow to improve upon the soft-collinear approximations made in the parton shower, such that hard radiation is simulated according to exact matrix elements. In the ME+PS approach [1] higher-order tree-level matrix elements for different final-state jet multiplicity are merged with each other and with subsequent parton shower emissions to generate an inclusive sample. Such a prescription is invaluable for analyses which are sensitive to final states with a large jet multiplicity. The only remaining deficiency of such tree-level calculations is the large uncertainty stemming from scale variations. The PowHEG method [2] solves this problem for the lowest multiplicity subprocess by combining full NLO matrix elements with the parton shower. While this leads to NLO accuracy in the inclusive cross section and the exact radiation pattern for the first emission, it fails to describe higher-order emissions with improved accuracy. Thus it is not sufficient if final states with high jet multiplicities are considered.

With the complementary advantages of these two approaches, the question arises naturally whether it would be possible to combine them into an even more powerful one. Such a combined algorithm was independently developed in [5] and [6]. Here a summary of the algorithm is given and predictions from corresponding Monte-Carlo predictions are presented.

\section{The MENLOPS formalism}

To combine both, the ME+PS and POWHEG method, one has to work out how they contribute to a cross section including up to the first emission off a given core process. All further emissions are not affected by the POWHEG approach, and can thus be treated as before in ME+PS.

The master formula for the expectation value of an observable in the POWHEG method contains two terms contributing to the cross section at NLO and can be written down in a simplified form (for details the reader is referred to [2], [4]):

$$
\langle O\rangle^{\mathrm{POW}}=\sum_{i} \int \mathrm{d} \Phi_{B} \overline{\mathrm{B}}_{i}\left(\Phi_{B}\right)[\underbrace{\bar{\Delta}_{i}\left(t_{0}\right) O\left(\Phi_{B}\right)}_{\text {no emission }}+\underbrace{\sum_{j} \int_{t_{0}} \mathrm{~d} \Phi_{R \mid B} \frac{\mathrm{R}_{j}\left(\Phi_{R}\right)}{\mathrm{B}_{i}\left(\Phi_{B}\right)} \bar{\Delta}_{i}(t) O\left(\Phi_{R}\right)}_{\text {resolved emission }}],
$$

where $\overline{\mathrm{B}}_{i}\left(\Phi_{B}\right)$ is the differential cross section at NLO for the Born phase-space configuration $\Phi_{B}$ and $\bar{\Delta}_{i}(t)=\exp \left\{-\sum_{j} \int_{t} \mathrm{~d} \Phi_{R \mid B} \mathrm{R}_{\mathrm{j}} / \mathrm{B}_{\mathrm{i}}\right\}$ is the matrix-element-corrected Sudakov form factor. The indices $i$ and $j$ label parton configurations, see [4]. The radiative phase space $\Phi_{R \mid B}$ is parametrised by variables in a parton-shower picture, including the ordering variable $t$ which is bounded from below by the cut-off $t_{0} \sim \Lambda_{\mathrm{QCD}}^{2}$, regularising the integral. 
The same quantity can be calculated in the ME+PS approach again only taking into account the first emission:

$$
\begin{aligned}
\langle O\rangle^{\mathrm{ME}+\mathrm{PS}}=\sum_{i} \int \mathrm{d} \Phi_{B} \mathrm{~B}_{i}\left(\Phi_{B}\right)[\underbrace{\Delta_{i}\left(t_{0}\right) O\left(\Phi_{B}\right)}_{\text {no emission }}+\sum_{j} \int_{t_{0}} \mathrm{~d} \Phi_{R \mid B} \\
\left.\times(\underbrace{\Theta\left(Q_{\text {cut }}-Q\right) \frac{8 \pi \alpha_{s}}{t} \mathscr{K}_{R_{j} \mid B_{i}} \frac{\mathscr{L}_{R_{j}}}{\mathscr{L}_{B_{i}}}}_{\text {PS domain }}+\underbrace{\Theta\left(Q-Q_{\text {cut }}\right) \frac{\mathrm{R}_{j}\left(\Phi_{R}\right)}{\mathrm{B}_{i}\left(\Phi_{B}\right)}}_{\text {ME domain }}) \Delta_{i}(t) O\left(\Phi_{R}\right)\right] .
\end{aligned}
$$

The term for resolved emissions is now separated into the "ME domain" and "PS domain" and describes the probability of additional QCD radiation according to the real-radiation matrix elements and their corresponding parton-shower approximations, respectively. $\mathscr{K}_{R_{j} \mid B_{i}}$ denote the evolution kernels of the parton shower and $\mathscr{L}_{R_{j} / B_{i}}$ are the parton luminosities of the real-emission and the underlying Born configurations. The Sudakov form factor $\Delta_{i}(t)$ is given by the parton shower and does not include matrix-element corrections, in contrast to $\bar{\Delta}_{i}(t)$ in Eq. (2.1).

The MENLOPS approach then combines the two above equations:

$$
\begin{aligned}
\langle O\rangle^{\mathrm{MENLOPS}}=\sum_{i} \int & \mathrm{d} \Phi_{B} \overline{\mathrm{B}}_{i}\left(\Phi_{B}\right)[\underbrace{\bar{\Delta}_{i}\left(t_{0}\right) O\left(\Phi_{B}\right)}_{\text {no emission }}+\sum_{j} \int_{t_{0}} \mathrm{~d} \Phi_{R \mid B} \frac{\mathrm{R}_{j}\left(\Phi_{R}\right)}{\mathrm{B}_{i}\left(\Phi_{B}\right)} \\
& \left.\times(\underbrace{\Theta\left(Q_{\text {cut }}-Q\right) \bar{\Delta}_{i}(t)}_{\text {PS domain }}+\underbrace{\Theta\left(Q-Q_{\text {cut }}\right) \Delta_{i}(t)}_{\text {ME domain }}) O\left(\Phi_{R}\right)\right] .
\end{aligned}
$$

The cross section of such a sample is determined by the NLO weight $\bar{B}$ as in POWHEG and could only be modified due to the appearance of $\Delta_{i}(t)$ instead of $\bar{\Delta}_{i}(t)$ in the "ME domain". Expanding the induced correction factor $\bar{\Delta}_{i}(t) / \Delta_{i}(t)$ to first order reveals that Eq. (2.3) automatically yields next-to-leading order accurate predictions for any infrared and collinear safe observable $O$. At the same time it becomes straightforward to include higher-order tree-level matrix elements to improve the description of high-multiplicity jet final states.

\section{Results}

The following collection of results showcases the improved description of experimental data as well as the significant difference to pure POWHEG samples due to the improved description of additional hard radiation. We employ the Monte-Carlo event generator SHERPA [3], which provides automated implementations of both ME+PS merging and the POWHEG algorithm as reformulated in [4]. Fig. 1 displays results for electron-positron annihilation into hadrons at $\sqrt{s}=91.25 \mathrm{GeV}$. In both, the jet resolution distribution for $5 \rightarrow 4$ clusterings in the Durham algorithm and the KSW angle between the four hardest jets, the improved description from exact matrix elements including all correlations is exemplified. Results for deep-inelastic lepton-nucleon scattering (DIS) are presented in Fig. 2. We show the pseudorapidity difference between the forward and backward jet in dijet events. Since these distributions are differential in the jet transverse energy in the Breit frame, they provide an excellent test of the correct simulation of jet activity in the Monte-Carlo. We observe a drastic difference between the POWHEG and MENLOPS predictions. Only the improved 

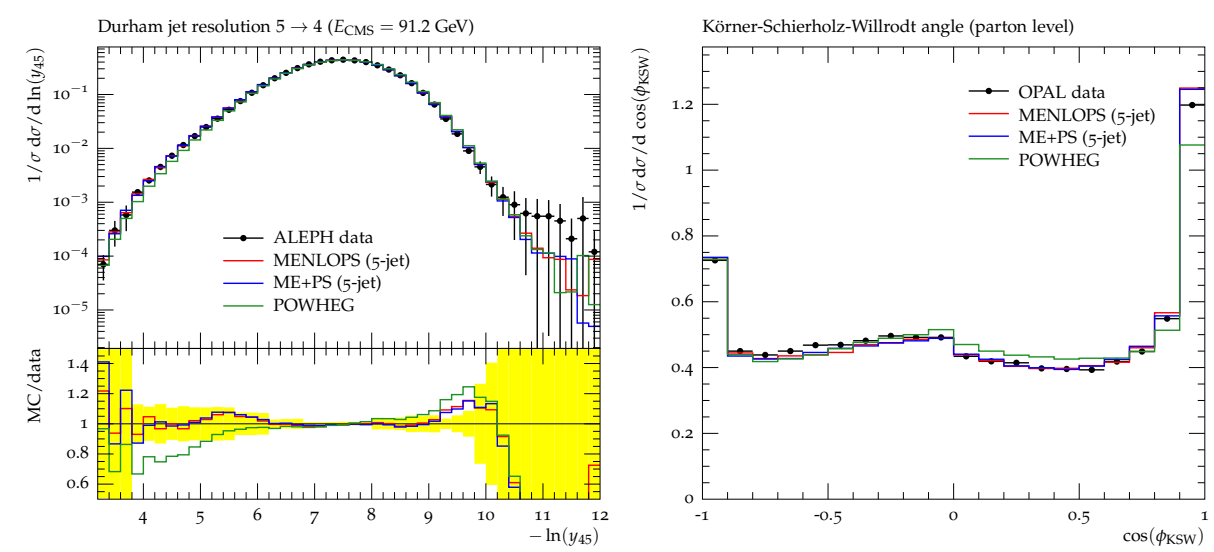

Figure 1: Jet resolution for $5 \rightarrow 4$ clusterings in the Durham algorithm (left) and KSW angle between the four hardest jets (right) in $e^{+} e^{-} \rightarrow$ hadrons at $\sqrt{s}=91.25 \mathrm{GeV}$.
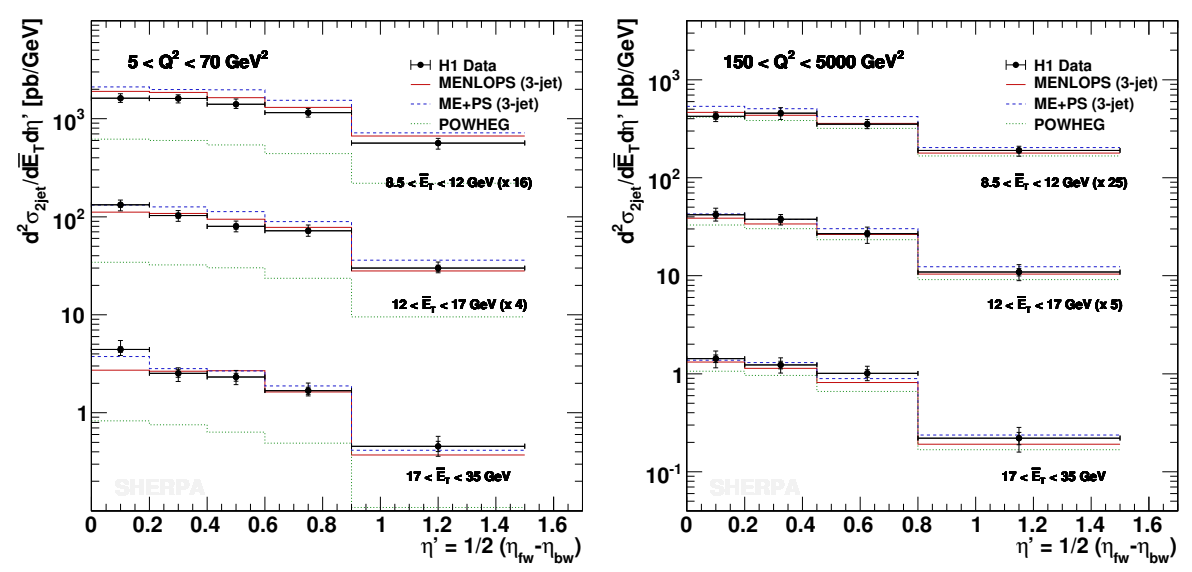

Figure 2: Pseudorapidity difference between the forward and backward jet in DIS dijet events for $e^{+} p \rightarrow$ $e^{+}+$jets at $\sqrt{s}=300 \mathrm{GeV}$.

simulation of additional hard QCD radiation through the MENLOPS approach allows to achieve agreement with experimental data. For jet production in association with a Drell-Yan lepton pair at Tevatron Run 2 energies one can find similar results in Fig. 3. Both, the inclusive jet multiplicity as well as the azimuthal separation of the lepton pair and the leading jet are described significantly better when higher-multiplicity matrix elements are included. Turning to predictions for the LHC, Fig. 4 shows the scalar sum of missing $E_{T}$ and transverse momenta of jets and leptons as well as the azimuthal decorrelation between the leading and second leading jet in $W^{+} W^{-}$production. Both observables are significantly influenced by final states with many jets, and thus show a strong difference between the POWHEG and MENLOPS results. For all processes investigated here, the results of the tree-level ME+PS approach resemble the features of the MENLOPS results as long as they are multiplied with a global $K$-factor as indicated in the plot legends. 

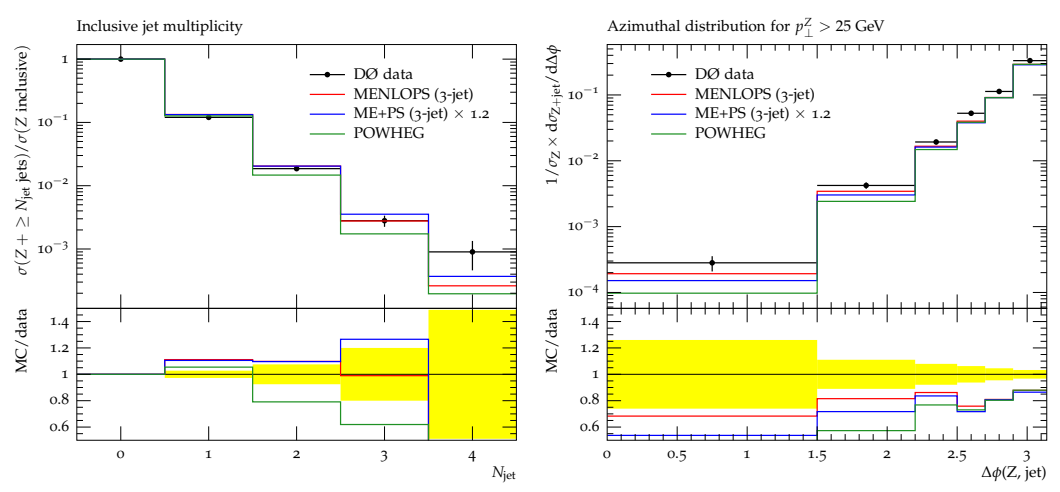

Figure 3: Inclusive jet multiplicity (left) and azimuthal separation of lepton pair and leading jet (right) in $\bar{p} p \rightarrow \ell \ell+$ jets at $\sqrt{s}=1.96 \mathrm{TeV}$
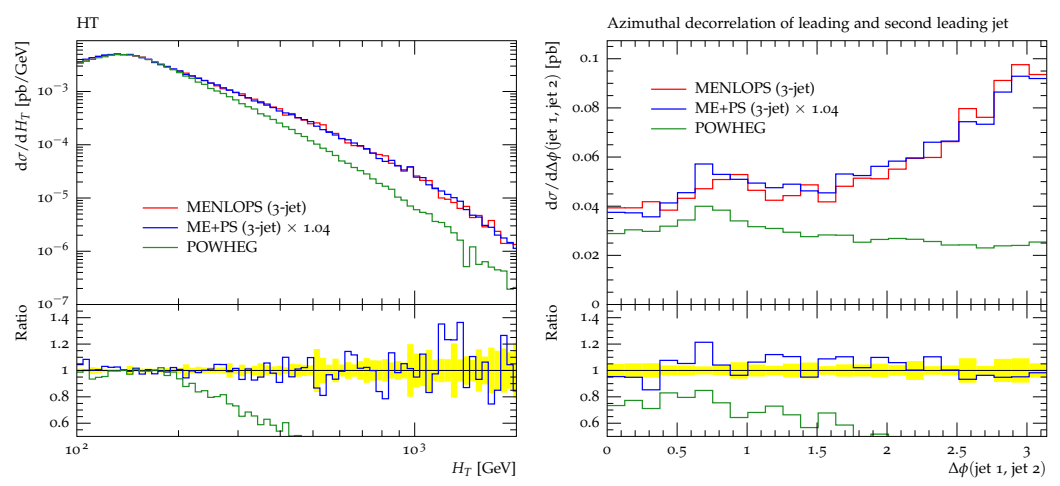

Figure 4: Scalar sum of missing $E_{T}$ and transverse momenta of jets and leptons (left) and azimuthal decorrelation between leading and second leading jet (right) in $p p \rightarrow W^{+} W^{-}+$jets at $\sqrt{s}=14 \mathrm{TeV}$

\section{References}

[1] S. Höche, F. Krauss, S. Schumann and F. Siegert, QCD matrix elements and truncated showers, JHEP 05 (2009), 053, [arXiv: 0903.1219 [hep-ph]].

[2] P. Nason, A new method for combining NLO QCD with shower Monte Carlo algorithms, JHEP 11 (2004), 040, [hep-ph/ 0409146 ]; S. Frixione, P. Nason and C. Oleari, Matching NLO QCD computations with parton shower simulations: the POWHEG method, JHEP 11 (2007), 070, [arXiv:0709.2092 [hep-ph]].

[3] T. Gleisberg, S. Höche, F. Krauss, A. Schälicke, S. Schumann and J. Winter, SHERPA 1. $\alpha, a$ proof-of-concept version, JHEP 02 (2004), 056, [hep-ph/ 0311263 ]; T. Gleisberg, S. Höche, F. Krauss, M. Schönherr, S. Schumann, F. Siegert and J. Winter, Event generation with SHERPA 1.1, JHEP 02 (2009), 007, [arXiv: 0811.4622 [hep-ph]].

[4] S. Höche, F. Krauss, M. Schönherr and F. Siegert, Automating the POWHEG method in SHERPA, arXiv: 1008.5399 [hep-ph].

[5] K. Hamilton and P. Nason, Improving NLO-parton shower matched simulations with higher order matrix elements, JHEP 06 (2010), 039, [arXiv: 1004.1764 [hep-ph]].

[6] S. Höche, F. Krauss, M. Schönherr and F. Siegert, NLO matrix elements and truncated showers, arXiv: 1009.1127 [hep-ph]. 